\title{
A New Online Resource to Monitor New or Emerging Plant Pests: MEDISYS Media Monitoring and the Case of Xylella fastidiosa
}

\author{
Franco Ferilli, ${ }^{1}$ Giuseppe Stancanelli, ${ }^{1}$ Jens P. Linge, ${ }^{2}$ and Maria Rosaria Mannino ${ }^{1, \dagger}$ \\ ${ }^{1}$ Animal and Plant Health Unit, European Food Safety Authority, Parma, Italy \\ ${ }^{2}$ Joint Research Centre, European Commission, Ispra, Italy
}

\begin{abstract}
The European Food Safety Authority has established a horizon scanning exercise for plant pests by automated monitoring of open-source media. The news sources are screened using the publicly accessible MEDISYS (Medical Information System) platform of the Joint Research Centre of the European Commission. Here, we report the example of monitoring for Xylella fastidiosa, a highly polyphagous plant-pathogenic bacterium. Since its first occurrence in Europe, news sources have reported findings and latest developments. Media monitoringrelated data can support surveillance or plant pests' management programs by early warning and can help understand the impacts of plant pests and the societal response to new plant health threats.
\end{abstract}

\section{Media Monitoring Platform Announcement}

In collaboration between the European Food Safety Authority (EFSA) and the European Commission Directorate-General Joint Research Centre, a media monitoring system for the surveillance of plant pests was set up; FAO defines a pest as "any species, strain or biotype of plant, animal or pathogenic agent injurious to plants or plant products" (FAO 2011). The system is based upon the fully automatic event-based surveillance system MEDISYS (Medical Information System).

MEDISYS is part of the Europe Media Monitor (EMM) system (Steinberger et al. 2013) and is a fully automatic public health surveillance system to monitor reporting on human and animal communicable diseases, chemical, biological, radiological and nuclear threats, and food and feed contaminations (Linge et al. 2009). The EMM system retrieves news items from official and unofficial media sites, general news media and selected blogs. Generally, news items are either retrieved from RSS feeds or by crawling HTML sites. RSS feeds, or rich site summary, are a type of web feed allowing the user to access and keep track of updated online content in a standard format.

Over 22,000 feeds of general news sites from over 107 countries are currently monitored. These sources comprise news websites at national, regional and local levels. In addition, feeds from specialist plant health sources, such as scientific journals, were added in the directory of monitored sources.

A Plant Health Threat Ontology with pest and disease names coming from multilingual sources such as UniProt Taxon (The UniProt Consortium 2016), European and Mediterranean Plant Protection Organization (EPPO) (EPPO 2018), and Wikipedia was built (Alomar et al. 2015, 2016). Multilingual keywords definitions for 560 categories for plant health threats, or

\footnotetext{
${ }^{\dagger}$ Corresponding author: M. R. Mannino; E-mail: alpha @ efsa.europa.eu
}

The positions and opinions presented in this article are those of the authors alone and are not intended to represent the views or any official positions of their institutions.

Accepted for publication 23 August 2018.

(C) 2019 The American Phytopathological Society 
Table 1. Number of news items selected by the Xylella fastidiosa category from February 2017 to March 2018 for the 10 most active countries

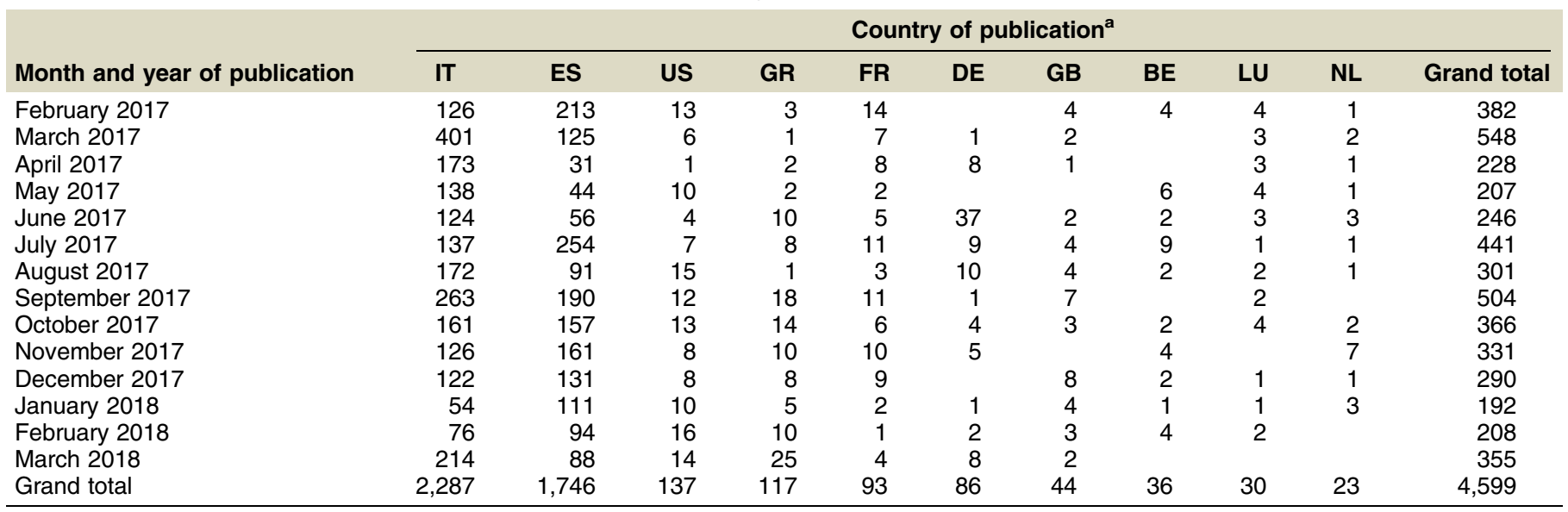

a Country of publication: IT = Italy, ES = Spain, US = United States, GR = Greece, FR = France, DE = Germany, GB = Great Britain, BE = Belgium, LU = Luxembourg, and NL = Netherlands.

pests, were created. Scientific and common names comprising colloquial terms used by journalists and the general public were used.

The system categorizes all incoming items according to the ontology of plant pests, identifies known names such as organizations, persons and locations, extracts events, clusters news items and calculates statistics to detect emerging threats. Each item thus comes with metadata (Alomar et al. 2016) that are added during the automated processing. The metadata include among others (i) entity, or people or organizations automatically recognized by EMM; (ii) category, or plant health threats as defined in the multilingual ontology; (iii) geolocation, or geographic locations mentioned in the items; and (iv) quote, or extract of text recognized by the processing chain as quotes.

Here, we present the monitoring results for the highly polyphagous plant-pathogenic bacterium Xylella fastidiosa (Wells et al. 1987) carried out in the period from February 2017 to March 2018.

The multilingual category definitions for $X$. fastidiosa keywords cover the scientific names, various associated disease names, and common names of the pathogen.

Raw data were filtered to show title of the news item, URL of the item, date of publication, and country of publication. Pivot table were created in Microsoft Excel to visualize and analyze the different trends over time and to identify peaks. These peaks were subsequently matched with specific events in the different countries in the news history. For example, a peak in the number of news items from Spain in July 2017 (Table 1) is due to the first findings on Spanish mainland of $X$. fastidiosa in almond orchards. The infected plants were identified in the province of Alicante, Valencian Community.

The data for the period from February 2017 to March 2018 show a high number of news stories about $X$. fastidiosa, with a total of 5,299 news items retrieved by the corresponding MEDISYS category. The news items come from a diverse group of 630 sources from 62 countries. This underlines the multilingual ability of EMM and of the developed ontology to retrieve news items. Table 1 details the number of news items selected for the different sources from the 10 most active countries. Most of the news items mention geolocations in Italy (2,287 items), Spain (1,746), United States (137), Greece (117), France (93), Germany (86), Great Britain (44), Belgium (36), Luxembourg (30), and the Netherlands (23).

The media monitoring shows that $X$. fastidiosa is a highly discussed topic, with many items referring to detection, prevention, and control of the pest in different European countries. The EFSA media monitoring results are regularly presented and discussed at the Plant Health section of the Committee on Plants, Animals, Food and Feed.

MEDISYS is publicly available; see http://medisys.newsbrief.eu/medisys/alertedition/en/ XylellaFastidiosa-PHT.html for the latest news about $X$. fastidiosa. Articles can be filtered by language, news sources, country of the news sources, and country mentioned in the text. In addition, the content retrieved for all 560 pest categories is available in the dedicated sections on the same webpage. A subscription to a customizable e-mail alert is available as well. Users 
can screen the categorized articles and display world maps highlighting event locations together with statistics on the reporting of plant health threats and countries.

A real-time map with event locations for articles retrieved by MEDISYS is available at http:// medisys.newsbrief.eu/emmMap/?type=category\&language=all\&category=XylellaFastidiosaPHT.

Media monitoring data can be used as a supplementary method to usual biosurveillance to help plant protection organizations with early warning, awareness, and rapid response (Thomas et al. 2011). Monitoring public media can thus support surveillance or pest management programs and can help understand the impacts of plant pests and the societal response to new plant health threats.

\section{Literature Cited}

Alomar, O., Batlle, A., Brunett, J. M., García, R., Gil, R., Granollers, T., Jiménez, S., Laviña, A., Linge, J. P., Pautasso, M., Reverté, C., Riudavets, J., Rortais, A., Stancanelli, G., Volani, S., and Vos, S. 2015. Development and testing of the media monitoring tool MedlSys for early identification and reporting of existing and emerging plant health threats. EPPO Bull. 45:288-293.

Alomar, O., Batlle, A., Brunett, J. M., García, R., Gil, R., Granollers, T., Jiménez, S., Laviña, A., Reverté, C., Riudavets, J., and Birgili-Gomá, J. 2016. Development and testing of the media monitoring tool MedlSys for the monitoring, early identification and reporting of existing and emerging plant health threats. EFSA Supporting Publications 2016:13(12):EN-1118.

EPPO. 2018. EPPO global database. European and Mediterranean Plant Protection Organization (EPPO). https://gd.eppo.int/

FAO. 2011. ISPM 5: Glossary of Phytosanitary Terms. International Plant Protection Convention. Food and Agriculture Organization of the United Nations (FAO).
Linge, J. P., Steinberger, R., Weber, T. P., Yangarber, R., van der Goot, E., Al Khudhairy, D. H., and Stilianakis, N. 2009. Internet surveillance systems for early alerting of health threats. Euro Surveill. 14:19162.

Steinberger, R., Pouliquen, B., and van der Goot, E. 2013. An introduction to the Europe Media Monitor family of applications. arXiv preprint arXiv:1309.5290.

The UniProt Consortium. 2016. UniProt: The universal protein knowledgebase. Nucleic Acids Res. 45:D158-D169.

Thomas, C. S., Nelson, N. P., Jahn, G. C., Niu, T., and Hartley, D. M. 2011. Use of media and public-domain Internet sources for detection and assessment of plant health threats. Emerg. Health Threats J. 4:7157.

Wells, J. M., Raju, B. C., Hung, H.-Y., Weisburg, W. G., Mandelco-Paul, L., and Brenner, D. J. 1987. Xylella fastidiosa gen. nov., sp. nov: Gram-negative, xylem-limited, fastidious plant bacteria related to Xanthomonas spp. Int. J. Syst. Evol. Microbiol. 37:136-143. 